Revue internationale de l'économie sociale

Recma

\title{
Economie territoriale et économie immatérielle : quels enjeux pour l'ESS ?
}

\section{Jean-François Draperi}

Numéro 337, juillet 2015

URI : https://id.erudit.org/iderudit/1032516ar

DOI : https://doi.org/10.7202/1032516ar

Aller au sommaire du numéro

Éditeur(s)

Association Recma

ISSN

1626-1682 (imprimé)

2261-2599 (numérique)

Découvrir la revue

Citer ce document

Draperi, J.-F. (2015). Economie territoriale et économie immatérielle : quels enjeux pour l'ESS ? Revue internationale de l'économie sociale, (337), 4-6.

https://doi.org/10.7202/1032516ar d'utilisation que vous pouvez consulter en ligne. 


\section{ECONOMIE TERRITORIALE ET ÉCONOMIE IMMATÉRIELLE : QUELS ENJEUX POUR L'ESS ?}

Nous savons désormais que l'économie sociale et solidaire (ESS) est capable, en complémentarité avec les collectivités territoriales et en partenariat avec l'économie de proximité des TPE et PME, de participer à une politique économique des territoires alternative à l'économie dominée par les grandes sociétés de capitaux.

\section{Vers une économie territoriale}

En France, comme dans de nombreux pays, des citoyens se réunissent en effet dans des associations ou des coopératives pour produire eux-mêmes leur énergie, distribuer l'eau, construire de nouveaux habitats, acquérir et exploiter des terres, échanger localement et équitablement, etc. L’engagement des collectivités territoriales - régions, départements, communes - se confirme chaque jour : hier, les collectivités offraient des zones industrielles à l'intention d'entrepreneurs extérieurs; aujourd'hui, elles s'engagent dans l'économie de leur territoire, introduisant par exemple des clauses sociales et environnementales dans leurs appels d'offres; demain (déjà aujourd'hui pour certaines, comme le Vorarlberg en Autriche), elles concevront des pans entiers de l'économie à partir des ressources locales.

Elles empruntent, souvent sans le savoir, la voie dans laquelle s'étaient engagés les Etats de l'hémisphère sud accédant à l'indépendance dans la décennie 1960 : celle d'un développement que l'on définissait alors comme autocentré ou encore « écodéveloppement » (Ignacy Sachs). Une révolution technologique nous sépare cependant de cette période. Aujourd'hui, ces initiatives se relient à d'autres avec lesquelles elles établissent des échanges matériels, par exemple dans le cadre du commerce équitable, ou des échanges immatériels, à partir des nouvelles technologies de l'information et de la communication. Et l'économie immatérielle est sans aucun doute l'un des domaines dans lesquels l'ESS doit le plus progresser dans les années à venir, sous peine que les économies territoriales se cantonnent dans les niches d'un capitalisme qui se déplace au rythme de la révolution technologique et de ses effets.

\section{L'économie immatérielle}

Les enjeux liés à l'économie immatérielle revêtent au moins trois aspects d'ordre technique, économique et social.

Le premier aspect, technique, de cette économie est qu'elle est moins immatérielle qu'elle ne paraît. Le fait qu'elle déplace les termes de l'échange vers l'usage à partir de la propriété n'efface en rien la réalité de celle-ci. Et cette propriété est liée à la maîtrise de la production de biens matériels qui vont 
du logiciel au satellite en passant par le téléphone et les médias. Le propriétaire dicte en partie les termes de l'échange et a le pouvoir de les transformer. Ce pouvoir est cependant très différent selon que l'échange fait appel ou non à un serveur central. Dans tous les cas, cependant, le propriétaire agit en fonction d'intérêts privés et financiers, et il rencontre en partie au moins les intérêts des usagers.

Deuxième aspect, économique: il n'y a pas de dématérialisation qui ne s'appuie sur des valeurs. La valeur universelle de l'échange dématérialisé tel qu'il est aujourd'hui le plus souvent pratiqué est essentiellement le gain personnel et généralement matériel. C'est parce que l'on y gagne, parfois en temps et toujours économiquement, et plus précisément financièrement, que l'on utilise les nouveaux médias de communication pour obtenir un bien ou un service. Il faut toutefois souligner que cette économie procède également d'un nouveau mode de production de la valeur par des modalités d'échanges inédites et de nouvelles formes d'organisation du travail. Dans sa dimension collaborative, l'économie immatérielle se caractérise par la mutualisation de moyens et l'organisation horizontale des usagers-citoyens en « réseaux » ouverts ou en " communautés ». Ses pratiques sont ainsi très variées et en évolution constante.

Troisième aspect, social: l'économie immatérielle met en question le lien social et des règles collectives anciennes. A travers Airbnb, Uber, BlaBlaCar, etc., cette économie met à mal l'exercice de métiers historiques - réservation directe, taxis, réseaux de transports collectifs - qui se sont souvent construits sur plusieurs décennies. C'est sinon la totalité, au moins la majorité des professions et des organisations de travail qui sont touchées: l'économie immatérielle peut inclure l'échange de logements, les consommations de tous types, la production culturelle, le covoiturage, le coworking (espace de travail partagé), le crowdfunding (financement participatif), les fablabs (« laboratoires de fabrication »), etc.

Celle-ci questionne simultanément les régulations garanties par les Etats dans la mesure où cette économie peut s'affranchir des conventions collectives et des lois nationales. En même temps, elle uniformise les pratiques économiques en les orientant toutes par rapport à un mode de gestion spécifique qui fait abstraction des normes sociales et culturelles locales et régionales.

\section{Une chance pour l'ESS}

Or, en dépit ou peut-être en raison de ces caractères, l'économie immatérielle est une chance pour l'économie sociale et solidaire. Elle l'est d'abord parce que l'économie immatérielle ne suppose pas des investissements aussi lourds que l'économie industrielle, investissements matériels dont la masse limite depuis un siècle et demi la capacité des coopératives à se hisser au niveau du pouvoir d'investissement des sociétés de capitaux. Elle l'est ensuite parce que l'économie immatérielle peut être aussi collaborative, donc associative, coopérative et mutualiste. Elle peut l'être d'autant plus qu'elle est aussi une économie de la connaissance dans laquelle l'économie sociale est à son avantage. La possibilité de définir des usages nouveaux rend possible l'adoption par les usagers de règles nouvelles. Il incombe à l'ESS 
de susciter et de définir des ensembles de règles d'usage des nouvelles technologies tournées vers la satisfaction de besoins collectifs.

Ce champ de pratiques est susceptible de constituer un espace où les forces respectives de l'économie sociale, de l'économie solidaire et de l'entrepreneuriat social peuvent s'allier et se conjuguer: la première en apportant à la fois l'expérience que la profondeur de son histoire lui a permis d'acquérir pensons par exemple aux sept principes coopératifs de l'Alliance coopérative internationale (ACI) - et la force matérielle de ses entreprises en termes de capital et de réseaux; la deuxième, par l'extension de la solidarité au plus grand nombre par de nouveaux moyens; le troisième, par sa capacité à se saisir des nouveaux espaces économiques ouverts par les nouvelles technologies de l'information et de la communication (NTIC).

Pas plus que l'économie industrielle, l'économie immatérielle n'est une fin en elle-même. Elle est aujourd'hui dominée par l'intérêt privé et les stratégies de quelques très grandes multinationales. A l'ESS de s'en saisir et de la mettre au service de la société en l'intégrant dans l'édification des économies territoriales et de l’inter-territorialité.

JEAN-FrançoIs DRAPERI 